\title{
Rate and Reliability Trade-off for mmWave Communication via Multi-point Connectivity
}

\author{
Dileep Kumar, Jarkko Kaleva, Antti Tölli \\ Centre for Wireless Communications, University of Oulu, Oulu, Finland \\ Email: \{dileep.kumar, jarkko.kaleva, antti.tolli\}@oulu.fi
}

\begin{abstract}
The fundamental challenge of mmWave frequency band is the sensitivity of the radio channel to blockage, which gives rise to unstable connectivity and also impacts the reliability of a system. In this paper, we explore the viability of using Coordinated Multi-point (CoMP) connectivity for robust and resilient downlink communication. We provide a novel iterative algorithm for Weighted Sum-Rate Maximization (WSRM) problem leveraging Successive Convex Approximation (SCA) with low computational complexity, which admits closed-form solution via iterative evaluation of optimal Karush-Kuhn-Tucker (KKT) conditions. Unlike in the conventional CoMP schemes, the propose precoder design has, per-iteration, computational complexity in the order of base-station (BS) antennas instead of system-wide joint transmit antennas. This is achieved by splitting the beamformer design to be parallel across the coordinating BSs. For the downlink precoder design, a conservative estimate of the user specific rate is obtained by considering possible combinations of potentially blocked links. In the presence of random blockages, the effective throughput and outage performance of the proposed method significantly outperform several baseline scenarios, hence providing more reliable connectivity.
\end{abstract}

\section{INTRODUCTION}

The proliferation of ever-increasing data-intensive wireless applications along with spectrum shortage motivates the investigation of millimeter-wave (mmWave) communication for 5th-generation (5G) and beyond cellular systems [1]-[3]. One of the fundamental challenges is the sensitivity of the mmWave radio channel to blockage due to reduced diffraction, higher path and penetration losses [3], which leads to rapid degradation of signal strength and gives rise to unstable connectivity. This problem is aggravated by the fact that, for instance, a human blocker can degrade the channel quality for up to hundreds of millisecond [3]. Such frequent and long duration blockage significantly reduces the quality-of-service.

To overcome such challenges, use of CoMP schemes, where the users are concurrently connected to multiple BSs, are imperative for more robust communication [4]-[8]. CoMP connectivity have been widely studied over past decade under the context of 4G systems [4], [5]. Recent studies have considered the deployment of CoMP in the mmWave frequencies [6][8], and proposed in 3GPP specifications as a key component for upcoming 5G systems [9]. CoMP techniques in [4]-[8] were mainly devised to enhance the capacity and coverage by

This work was supported by the Academy of Finland under grants no. 313041 (PRISMA: Positioning-aided Reliably-connected Industrial Systems with Mobile mmWave Access) and 318927 (6Genesis Flagship). utilizing the spatially separated transceivers. Thus, they were not originally designed for the stringent latency and reliability requirements of industrial-grade critical applications.

Motivated by the above concern, there is a need for more robust and resilient algorithms that can retain stable connectivity under the uncertainties of mmWave channel and random mobile blockers. To this end, we provide a novel beamformer design for WSRM with a strong emphasis on system-level reliability. We consider a conservative estimate of the available rate over all possible combinations of potentially blocked links among the cooperating BSs. Managing large set of link blockage combinations is considerably more difficult than conventional quality-of-service constrained optimization [10] due to the mutually coupled Signal-to-Interference-Plus-Noise Ratio (SINR) constraints. We provide a novel low-complexity SCA algorithm for the original non-convex and computationally challenging problem. The proposed framework merges the dual [11] and best response [12] decomposition methods to admit parallel beamformer processing across the distributed BS antennas via iterative evaluation of the closed-form KKT conditions. The schemes proposed in [10], [12] cannot be used directly, thus our proposed KKT based solution is significantly more advanced, and provides novel approach for solving highly coupled minimum SINR constraints. This provides a practical, latency-conscious and computationally efficient implementation for cloud edge clusters. Finally, we quantify the complexity and performance advantages of this solution in terms of sum-rate and reliable connectivity.

\section{SySTEM MODEL}

We consider downlink in a mmWave based multi-user multiple-input single-output (MU-MISO) communication system, consisting of $K$ single antenna users served by $B$ BSs. Each BS is equipped with $N_{t}$ transmit antennas, arranged in a uniform linear array (ULA) pattern with $s=\lambda / 2$ spacing between any two adjacent antenna elements, where $\lambda$ is the wavelength of carrier frequency. We define $\mathcal{B}=\{1,2, \ldots, B\}$ to be the set of all serving BSs, $\mathcal{K}=\{1,2, \ldots, K\}$ denotes the set active users, and serving set of BSs for user $k$ is represented with $\mathcal{B}_{k}$, where $\mathcal{B}_{k} \subseteq \mathcal{B}$. In this system model, $N_{t} \geq K$ i.e., all users can be spatially multiplexed. We consider Joint Transmission (JT)-CoMP, whereby, each active user $k$ receives a coherently synchronous signal from serving set of BSs $\mathcal{B}_{k}$. Further, all the downlink transmis- 


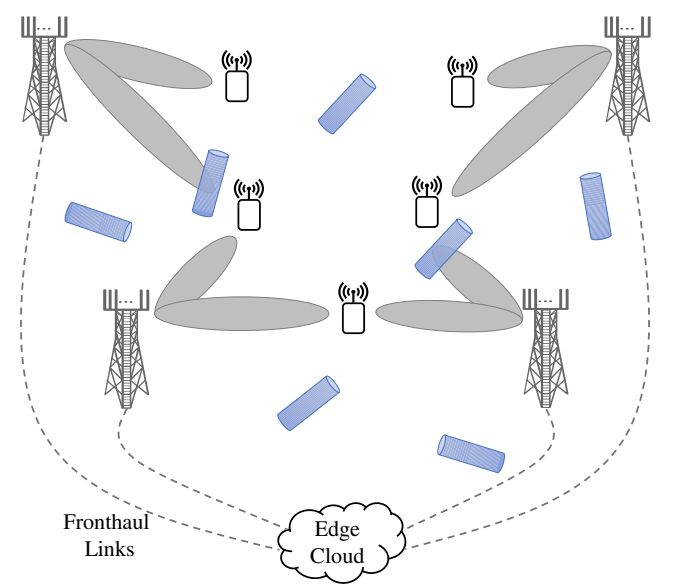

Fig. 1. Downlink communication system model.

sion are performed using same frequency and time channel. Furthermore, in this paper, we restrict ourselves to the case where each antenna is equipped with a dedicated baseband circuit to enable fully digital signal processing. Finally, we assume cloud (or centralized) radio access network (C-RAN), wherein all BSs are connected to the edge cloud by low-latency fronthaul links. It should be noted, in the C-RAN architecture, a common baseband processing unit (BBU) performs all the digital signal processing functionalities while BS implements limited radio operations [13]. Therefore, such fully centralized baseband processing provides more efficient BSs coordination, and thus enables effective implementation for JT-CoMP [13].

The received signal of $k^{t h}$ user, $y_{k}$ is defined as

$$
y_{k}=\sum_{b \in \mathcal{B}_{k}} \mathbf{h}_{b, k}^{\mathrm{H}} \mathbf{f}_{b, k} x_{k}+\sum_{k^{\prime} \in \mathcal{K} \backslash k} \sum_{b \in \mathcal{B}_{k^{\prime}}} \mathbf{h}_{b, k}^{\mathrm{H}} \mathbf{f}_{b, k^{\prime}} x_{k^{\prime}}+w_{k},
$$

where $w_{k} \in \mathcal{C N}\left(0, N_{o}\right)$ is circularly symmetric additive white Gaussian noise (AWGN) with power spectral density (PSD) of $N_{o}$ and $x_{k}$ is normalized data symbol, i.e., $\mathbb{E}\left\{\left|x_{k}\right|\right\}=1$. Finally, $\mathbf{f}_{b, k} \in \mathbb{C}^{N_{t} \times 1}$ represents the portion of the joint precoder between a BS-user pair $(b, k)$, designed by the BBU assuming perfect estimation of available (non-blocked) CSI. The received SINR for each user $k$ can be formulated as

$$
\gamma_{k}\left(\mathcal{B}_{k}\right)=\frac{\left|\sum_{b \in \mathcal{B}_{k}} \mathbf{h}_{b, k}^{\mathrm{H}} \mathbf{f}_{b, k}\right|^{2}}{N_{o}+\sum_{k^{\prime} \in \mathcal{K} \backslash k}\left|\sum_{b \in \mathcal{B}_{k^{\prime}}} \mathbf{h}_{b, k}^{\mathrm{H}} \mathbf{f}_{b, k^{\prime}}\right|^{2}} .
$$

\section{A. Channel \& Blockage Model}

Due to higher penetration and path losses, low-scattering and reduced diffraction at the mmWave frequencies, channel is spatially sparse [1], [3], where line-of-sight (LoS) link is the dominant path and mainly contributes to the communications [3], [6], [7]. Thus, unblocked LoS link is highly desirable to initiate and maintain stable mmWave communications [14]. The design of channel is based on sparse geometric model using extended Saleh-Valenzuela model, which is widely adapted for mmWave signal processing techniques. Specifically, we consider $M_{b, k}$ paths for the channel $\mathbf{h}_{b, k}$ between a BS-user pair $(b, k)$, and expressed as

$$
\mathbf{h}_{b, k}=\sqrt{\frac{N_{t}}{M_{b, k}}}\left\{g_{b, k}^{1} \mathbf{a}_{T}^{\mathrm{H}}\left(\phi_{b, k}^{1}\right)+\sum_{m=2}^{M_{b, k}} g_{b, k}^{m} \mathbf{a}_{T}^{\mathrm{H}}\left(\phi_{b, k}^{m}\right)\right\},
$$

where $\phi_{b, k}^{1}\left(\phi_{b, k}^{m}\right)$ denotes the angle-of-departure (AoD) for a LoS (NLoS) path. Note that the AoD for each NLoS path $m$ is assumed to be uniformly distributed $\phi_{b, k}^{m} \in[0,2 \pi]$, whereas, $\phi_{b, k}^{1}$ is related to actual position of BS-user $(b, k)$ pair [15]. The transmit array steering vector of ULA is $\mathbf{a}_{T}(\phi) \in \mathbb{C}^{N_{t} \mathrm{x} 1}$. Finally, $g_{b, k}^{1}=v_{b, k} d_{b, k}^{-\varrho}$ and $g_{b, k}^{m}=v_{b, k} d_{b, k}^{-\eta}$, in which $d_{b, k}$ is the BS-user distance, $v_{b, k}$ is zero mean random complex gain with unit variance and $\varrho(\eta)$ denotes the path-loss exponent for LoS (NLoS) link. By experimental results, $\eta$ is much higher than LoS path-loss exponent [3], [6], [7].

We consider a blockage model, where each link undergoes independent blocking. This is reasonably accurate (especially when the blockers are not too large) [14]. We simply assume blockage for the dominant LoS path while NLoS links are unobstructed. More specifically, the channel between any typical BS-user pair can have one of two states: fully-available or NLoS state. The NLoS state occurs when the dominant LoS link is blocked by any obstacle. Furthermore, even a mobile human blocker causes $30 \mathrm{~dB}$ attenuation and can obstruct the LoS path for hundreds of millisecond [3]. This can be equivalently modeled as $0^{N_{t} \times 1}$. The fully-available state is defined in (3).

Since blockers are completely random, their position and orientation may change multiple times within the channel coherence interval. Therefore, in general, blockage event and position of each blocker can not be known a priori in a dynamic mobile environment. We assume that the transmit beamformer at the BBU is designed assuming fully-available channel (3). Thus, a system can be in outage, if dominant LoS link is not available during the transmission, due to the random blockage. It should be noted, a LoS link can also be in the blockage state during the estimation phase and available during data transmission. However, that will not be included for the transmission. Thus, from the reliability prospective, we have to consider the case when channel is available during the estimation phase but not during the transmission, which is not a priori known at the $\mathrm{BBU}$.

\section{B. Problem Formulation}

The major goal of this work is to develop a robust and resilient donwlink transmission strategy that can retain a stable connectivity under the uncertainties of mmWave channel and random link blockage. To do that, we need to compute the optimal joint transmit beamformer $\mathbf{F}=\left[\mathbf{f}_{1,1}, \mathbf{f}_{1,2}, \ldots, \mathbf{f}_{B, K}\right]$, while emphasising the system-level reliability by exploiting multi-antenna spatial diversity with the CoMP connectivity. The precoder design can be formulated as $\mathrm{WSRM}^{1}$ problem as

$$
\begin{array}{cl}
\underset{\mathbf{F}, \tilde{\gamma}_{k}}{\operatorname{maximize}} & \sum_{k \in \mathcal{K}} \delta_{k} \log \left(1+\tilde{\gamma}_{k}\right) \\
\text { subject to } & \gamma_{k}\left(\mathcal{B}_{k}^{\mathrm{c}}\right) \geq \tilde{\gamma}_{k} \quad \forall k, \forall \mathcal{B}_{k}^{\mathrm{c}} \subseteq \mathcal{B}_{k},\left|\mathcal{B}_{k}^{\mathrm{c}}\right| \geq L, \\
& \sum_{k \in \mathcal{K}}\left\|\mathbf{f}_{b, k}\right\|^{2} \leq P_{b} \quad \forall b
\end{array}
$$

\footnotetext{
${ }^{1}$ In our model, we assume Gaussian signalling as the upper bound for the achievable rate expressions.
} 
where $\delta_{k} \geq 0 \forall k$ denotes the user-specific priority weights and $\gamma_{k}\left(\mathcal{B}_{k}^{\mathrm{c}}\right)$ is defined as in (2). Constraint (4c) limits the total transmit power for $b^{\text {th }}$ BS. Finally, constraint (4b) is the lower estimate of available SINR for each user $k$, which is computed over all subset combinations of size $\left|\mathcal{B}_{k}^{\mathrm{c}}\right| \geq L$ of serving set of BSs $\left(\mathcal{B}_{k}^{\mathrm{c}} \subseteq \mathcal{B}_{k}\right)$.

The resulting problem (4) is highly non-convex and intractable due a potentially large number of combinations of non-convex SINR constraint. Specifically, $\gamma_{k}\left(\mathcal{B}_{k}^{c}\right)$ cannot be solved directly in (4b) as a convex constraint. In Section IV, we provide a practical and computationally efficient iterative algorithm by exploiting the convex approximation techniques.

\section{REliABILITY AND ThROUGHPUT TRADE-OFF}

We assume blockers are uncorrelated and randomly distributed, thus, position of each blocker and/or blockage event is completely unknown. Therefore, to maintain a reliable connectivity under the uncertainties of mmWave channel, BBU models the SINR over all possible subset combination of potentially blocked links and uses the worst case estimate in the objective $\tilde{\gamma}_{k}$ for the beamformer design. For example, referring to constraint (4b) and Fig. 1, let $L=3$ and $\left|\mathcal{B}_{k}\right|=4$, then the subset $\overline{\mathcal{B}}_{k}$ includes the following BS indices

$$
\overline{\mathcal{B}}_{k}=\{\{1,2,3\},\{1,2,4\},\{1,3,4\},\{2,3,4\},\{1,2,3,4\}\} .
$$

Consequently, at the optimal solution $\tilde{\gamma}_{k}=\min \left(\gamma_{k}\left(\mathcal{B}_{k}^{\mathrm{c}}\right)\right)$, where $c \in \mathcal{C}=\left[1,2, \ldots, \sum_{l=L}^{\left|\mathcal{B}_{k}\right|}\left(\begin{array}{c}\left|\mathcal{B}_{k}\right| \\ l\end{array}\right)\right]$. Therefore, a reliable connectivity for each user $k$ can be guaranteed, even if, $\left|\mathcal{B}_{k}\right|-L$ dominant links are not available during the transmission phase. In contrary, if more than $L$ links were available, the actual achievable rate would be somewhat larger than the assigned rate.

We can observe the impact of Constraint (4b) on reliability and achievable sum-rate. For example, by using the smaller subset size $L$, we can improve the system reliability. However, it leads to lower SINR and hence lower rate to each user. Conversely, larger subset size $L$ can provide higher SINR and rate, but it is more susceptible to outage resulting in less stable connectivity for each user. Clearly, there is a trade-off between achievable sum-rate and reliable connectivity.

\section{PRECODER DESIGN}

In this section, we elaborate on finding an asymptotically optimal solution to (4), which is highly non-convex due to the SINR expression in (2). We employ a widely used convex approximation technique based on SCA framework [12], [16], [17], wherein all non-convex constraints are approximated with a sequence of convex approximations. The underlying approximate subproblem is then iteratively solved until the convergence. The SINR expression in (4b) can be relaxed as

$$
\begin{aligned}
\tilde{\gamma}_{k} & \leq \gamma_{k}\left(\mathcal{B}_{k}^{\mathrm{c}}\right) \triangleq \frac{\left|\sum_{b \in \mathcal{B}_{k}^{\mathrm{c}}} \mathbf{h}_{b, k}^{\mathrm{H}} \mathbf{f}_{b, k}\right|^{2}}{I_{k}\left(\mathcal{B}_{k}^{\mathrm{c}}\right)} \\
I_{k}\left(\mathcal{B}_{k}^{\mathrm{c}}\right) & \triangleq N_{o}+\sum_{k^{\prime} \in \mathcal{K} \backslash k}\left|\sum_{b \in \mathcal{B}_{k}^{\mathrm{c}}} \mathbf{h}_{b, k}^{\mathrm{H}} \mathbf{f}_{b, k^{\prime}}\right|^{2}
\end{aligned}
$$

where (5) is the lower bound for SINR and (6) is the total interference plus noise in (2). It should be noted that each subset combination $c$ in (4) will have a unique interference constraint, which includes all the interference terms corresponding to that BSs combination. This greatly simplifies the implementation of the iterative algorithm introduced later in this section. For conciseness, we rewrite (5) as

$$
\begin{aligned}
1+\tilde{\gamma}_{k} & \leq \frac{N_{o}+\sum_{i \in \mathcal{K}}\left|\sum_{b \in \mathcal{B}_{i}^{\mathrm{c}}} \mathbf{h}_{b, k}^{\mathrm{H}} \mathbf{f}_{b, i}\right|^{2}}{I_{k}\left(\mathcal{B}_{k}^{\mathrm{c}}\right)}, \\
I_{k}\left(\mathcal{B}_{k}^{\mathrm{c}}\right) & \leq \frac{N_{o}+\sum_{i \in \mathcal{K}}\left|\overline{\mathbf{h}}_{k}^{\mathrm{cH}} \overline{\mathbf{f}}_{i}\right|^{2}}{1+\tilde{\gamma}_{k}},
\end{aligned}
$$

where $\overline{\mathbf{f}}_{k} \in \mathbb{C}^{\left|\mathcal{B}_{k}\right| N_{t} \times 1}$ is the stacked transmit beamformer, $\overline{\mathbf{h}}_{k} \in \mathbb{C}^{\left|\mathcal{B}_{k}\right| N_{t} \times 1}$ is the stacked channel vector, defined as

$$
\begin{aligned}
\overline{\mathbf{f}}_{k} & \triangleq\left[\mathbf{f}_{\mathcal{B}_{k}(1), k}^{\mathrm{T}}, \mathbf{f}_{\mathcal{B}_{k}(2), k}^{\mathrm{T}}, \ldots, \mathbf{f}_{\mathcal{B}_{k}\left(\left|\mathcal{B}_{k}\right|\right), k}^{\mathrm{T}}\right]^{\mathrm{T}}, \\
\overline{\mathbf{h}}_{k} & \triangleq\left[\mathbf{h}_{\mathcal{B}_{k}(1), k}, \mathbf{h}_{\mathcal{B}_{k}(2), k}, \ldots, \mathbf{h}_{\mathcal{B}_{k}\left(\left|\mathcal{B}_{k}\right|\right), k}\right] .
\end{aligned}
$$

Finally, we denote the stacked channel for user $k$ corresponding to each subset combination as $\overline{\mathbf{h}}_{k}^{\mathrm{c}} \forall c$. For example, again referring to Fig. 1, let $L=3,\left|\mathcal{B}_{k}\right|=4$ and $\mathcal{B}_{k}^{c}=\{1,2,4\}$ then the corresponding stacked channel $\overline{\mathbf{h}}_{k}^{c} \triangleq\left[\mathbf{h}_{1, k}, \mathbf{h}_{2, k}, \mathbf{0}, \mathbf{h}_{4, k}\right]$.

Even with this relaxation of SINR in (2), by (8), (4) is still non-convex due to quadratic-over-linear constraint (8) and that cannot be handled directly [16]. Here, we resort to SCA framework, wherein all non-convex SINR constraints in (8) are approximated with convex subset and solved until the convergence [12], [16], [17]. We note that the RHS of (8) is a convex function, and thus can be bounded by the first order Taylor approximation as

$$
\begin{gathered}
\mathcal{F}\left(c, \overline{\mathbf{f}}_{k}, \tilde{\gamma}_{k} ; \overline{\mathbf{f}}_{k}^{(i)}, \tilde{\gamma}_{k}^{(i)}\right) \triangleq 2 \frac{\overline{\mathbf{f}}_{k}^{(i) \mathrm{H}} \overline{\mathbf{h}}_{k}^{\mathrm{c}} \overline{\mathbf{h}}_{k}^{\mathrm{cH}}}{1+\tilde{\gamma}_{k}^{(i)}}\left(\overline{\mathbf{f}}_{k}-\overline{\mathbf{f}}_{k}^{(i)}\right) \\
+\frac{N_{o}+\sum_{i \in \mathcal{K}}\left|\overline{\mathbf{h}}_{k}^{\mathrm{cH}} \overline{\mathbf{f}}_{i}^{(i)}\right|^{2}}{1+\tilde{\gamma}_{k}^{(i)}}\left(1-\frac{\tilde{\gamma}_{k}-\tilde{\gamma}_{k}^{(i)}}{1+\tilde{\gamma}_{k}^{(i)}}\right) \\
\leq \frac{N_{o}+\sum_{i \in \mathcal{K}}\left|\overline{\mathbf{h}}_{k}^{\mathrm{cH}} \overline{\mathbf{f}}_{i}\right|^{2}}{1+\tilde{\gamma}_{k}},
\end{gathered}
$$

where $\mathcal{F}\left(c, \overline{\mathbf{f}}_{k}, \tilde{\gamma}_{k} ; \overline{\mathbf{f}}_{k}^{(i)}, \tilde{\gamma}_{k}^{(i)}\right)$ is the under-estimator for the RHS of (8), with equality, only at the operating points $\left\{\overline{\mathbf{f}}_{k}^{(i)}, \tilde{\gamma}_{k}^{(i)}\right\}$. After replacing Constraint (4b) with LHS of (11), an approximate subproblem, for $i^{t h}$ SCA iteration, is expressed in convex form as

$$
\begin{array}{cl}
\underset{\mathbf{F}, \tilde{\gamma}_{k}}{\operatorname{maximize}} & \sum_{k \in \mathcal{K}} \delta_{k} \log \left(1+\tilde{\gamma}_{k}\right) \\
\text { subject to } & \mathcal{F}\left(c, \overline{\mathbf{f}}_{k}, \tilde{\gamma}_{k} ; \overline{\mathbf{f}}_{k}^{(i)}, \tilde{\gamma}_{k}^{(i)}\right) \geq I_{k}\left(\mathcal{B}_{k}^{\mathrm{c}}\right) \\
& \forall k, \forall \mathcal{B}_{k}^{\mathrm{c}} \subseteq \mathcal{B}_{k},\left|\mathcal{B}_{k}^{\mathrm{c}}\right| \geq L, \\
& \sum_{k \in \mathcal{K}}\left\|\mathbf{f}_{b, k}\right\|^{2} \leq P_{b} \quad \forall b .
\end{array}
$$

Problem (12) can be more efficiently solved by parallel beamformer updates across the distributed BSs antenna heads. Iterative evaluation of the KKT optimality conditions for each SCA step reveals conveniently parallel structure for the 


$$
\begin{aligned}
& \mathcal{L}\left(\mathbf{F}, \tilde{\gamma}_{k}, a_{k, c}, z_{b}\right)=-\sum_{k \in \mathcal{K}} \delta_{k} \log \left(1+\tilde{\gamma}_{k}\right)+\sum_{b \in \mathcal{B}} z_{b}\left(\sum_{k \in \mathcal{K}}\left\|\mathbf{f}_{b, k}\right\|^{2}-P_{b}\right) \\
& +\sum_{k \in \mathcal{K}} \sum_{c \in \mathcal{C}} a_{k, c}\left(N_{o}+\sum_{k^{\prime} \in \mathcal{K} \backslash k}\left|\overline{\mathbf{h}}_{k}^{\mathrm{cH}} \overline{\mathbf{f}}_{k^{\prime}}\right|^{2}-2 \frac{\overline{\mathbf{f}}_{k}^{(i) \mathrm{H}} \overline{\mathbf{h}}_{k}^{\mathrm{c}} \overline{\mathbf{h}}_{k}^{\mathrm{cH}}}{1+\tilde{\gamma}_{k}^{(i)}}\left(\overline{\mathbf{f}}_{k}-\overline{\mathbf{f}}_{k}^{(i)}\right)-\frac{N_{o}+\sum_{i \in \mathcal{K}}\left|\overline{\mathbf{h}}_{k}^{\mathrm{cH}} \overline{\mathbf{f}}_{i}^{(i)}\right|^{2}}{1+\tilde{\gamma}_{k}^{(i)}}\left(1-\frac{\tilde{\gamma}_{k}-\tilde{\gamma}_{k}^{(i)}}{1+\tilde{\gamma}_{k}^{(i)}}\right)\right) .
\end{aligned}
$$

beamformer design with significantly lower computational complexity w.r.t. joint beamformer optimization across all distributed BS antennas. The KKT based solution provides closed-form steps for an algorithm that does not rely on any generic convex solvers.

The Lagrangian $\mathcal{L}\left(\mathbf{F}, \tilde{\gamma}_{k}, a_{k, c}, z_{b}\right)$ of (12) is given in (13). The optimal solution can be obtained by iteratively solving a system of KKT conditions (for more detailed derivation see Appendix A). In (12b), the user specific SINR constraints are coupled over the link blockage combinations. This makes deriving an efficient solution via the KKT conditions considerably more difficult versus the case with only single SINR constraint per user [10], [12]. In the following, we provide a novel iterative algorithm by combining the SCA framework, which admits closed-form solution in each step. Specifically, the approximate convex subproblem is solved via the iterative evaluation of optimal KKT conditions. Furthermore, to improve the rate of convergence, the SCA approximation points $\mathbf{f}_{b, k}^{(i)}$ and $\tilde{\gamma}_{k}^{(i)}$ are also updated in each iteration along with other dual variables. To summarize, the steps in the iterative algorithm are

$$
\begin{aligned}
& \mathbf{f}_{b, k}^{*}=\left(\mathbb{I} z_{b}+\sum_{c \in \mathcal{C}} \sum_{k^{\prime} \in \mathcal{K} \backslash k} a_{k^{\prime}, c}^{(i-1)} \mathbf{h}_{b, k^{\prime}}^{\mathrm{c}} \mathbf{h}_{b, k^{\prime}}^{\mathrm{cH}}\right)^{-1} \mathbf{t}_{b, k}^{(i-1)}, \\
& \mathbf{f}_{b, k}^{(i)}=\mathbf{f}_{b, k}^{(i-1)}+\psi\left[\mathbf{f}_{b, k}^{*}-\mathbf{f}_{b, k}^{(i-1)}\right], \\
& \gamma_{k}^{(i)}\left(\mathcal{B}_{k}^{\mathrm{c}}\right)=\frac{\left|\overline{\mathbf{h}}_{k}^{\mathrm{cH}} \overline{\mathbf{f}}_{k}^{(i)}\right|^{2}}{N_{o}+\sum_{k^{\prime} \in \mathcal{K} \backslash k}\left|\overline{\mathbf{h}}_{k}^{\mathrm{cH}} \overline{\mathbf{f}}_{k^{\prime}}^{(i)}\right|^{2}}, \\
& \tilde{\gamma}_{k}^{(i)}=\delta_{k}\left\{\sum_{c \in \mathcal{C}} a_{k, c}^{(i-1)} \frac{N_{o}+\sum_{i \in \mathcal{K}}\left|\overline{\mathbf{h}}_{k}^{\mathrm{cH}} \overline{\mathbf{f}}_{i}^{(i-1)}\right|^{2}}{\left(1+\tilde{\gamma}_{k}^{(i-1)}\right)^{2}}\right\}^{-1}-1,
\end{aligned}
$$$$
a_{k, c}^{(i)}=\left(a_{k, c}^{(i-1)}+\beta\left[\tilde{\gamma}_{k}^{(i)}-\gamma_{k}^{(i)}\left(\mathcal{B}_{k}^{\mathrm{c}}\right)\right]\right)^{+}
$$

where

$$
\begin{aligned}
\mathbf{t}_{b, k}^{(i-1)}= & \left\{\sum_{c \in \mathcal{C}} a_{k, c}^{(i-1)} \frac{\left(\overline{\mathbf{f}}_{k}^{(i-1) \mathrm{H}} \overline{\mathbf{h}}_{k}^{\mathrm{c}}\right)}{1+\tilde{\gamma}_{k}^{(i-1)}} \mathbf{h}_{b, k}^{\mathrm{cH}}\right. \\
& \left.-\sum_{c \in \mathcal{C}} \sum_{k^{\prime} \in \mathcal{K} \backslash k} a_{k^{\prime}, c}^{(i-1)}\left(\sum_{b^{\prime} \in \mathcal{B} \backslash b} \mathbf{f}_{b^{\prime}, k}^{(i-1) \mathrm{H}} \mathbf{h}_{b^{\prime}, k^{\prime}}^{\mathrm{c}} \mathbf{h}_{b, k^{\prime}}^{\mathrm{cH}}\right)\right\},
\end{aligned}
$$

$\psi>0$ and $\beta>0$ are small positive step sizes, and $(x)^{+} \triangleq \max (0, x)$. Thus, the KKT expressions in (14) are solved in an iterative manner, starting with initializing the variables to some feasible values, such that SINR and total transmit power constraint for each distributed BS antenna head is satisfied. Finally, the beamformers design has been summarized in Algorithm 1.

The optimal precoder $\mathbf{F}$ is inherently coupled between all distributed BS antennas (12), because of the coherent joint transmission to each user. One possible approach is based on updating the beamformer sequentially i.e., using Gauss-Seidel type update process, which provides monotonic converges for a WSRM optimization problems [18]. Here, instead, we implement a parallel optimization framework which efficiently parallelizes the beamformer updates across the distributed BS antennas and hence significantly reduces the per-iteration computational complexity. For a given iteration, BS specific beamformers are solved in parallel while assuming the coupling from other BSs fixed and that is matched to the signal from the previous iteration (14a). The objective function can be shown to converge if we allow sufficiently large number of subgradient iterations per fixed SCA approximation (until increased objective) for each BS before making the best response step and with sufficiently small step sizes [12]. However, here we are more interested in fast and robust rate of convergence, for which we allow only a single subgradient update per best response iteration. It is shown by numerical examples that this provides good performance with practical number of iterations. It should be noted that the transmit power constraints are convex, therefore, regularized update with $\psi \leq$ 1 will strictly preserve the feasibility of total transmit power. Furthermore, dimensions of the inverse operation in (14a) is directly proportional to number of transmit antennas $\left(N_{t}\right)$ at each BS. In addition, complexity of the matrix inversion operation in (14a) can be alleviated by solving $\mathbf{f}_{b, k}^{*}$ from a system of linear equations.

Problem (12) can be solved in a generic convex optimization solver as a sequence of second-order cone programs (SOCP) [19], [20]. The complexity of the SINR constraints scales exponentially with the length of the joint beamformers $\left(B N_{t}\right)$ [20]. Thus, particularly, for dense mmWave deployments with large $N_{t}$ and $B$, the complexity quickly becomes intractable in practice. The proposed KKT solution is bounded by (14a), which scales with $N_{t}$. Thus, it provides a significant reduction in complexity for even modestly sized systems.

\section{Simulation Results}

We consider a downlink mmWave MU-MISO system with $B=K=4$, where the BSs are equipped with ULAs of $N_{t}=16$ antennas. Each BS is placed at the corner of a square cell with the inter-site spacing of 100 meters and connected to common BBU in the edge cloud. We consider JT-CoMP with full-coordination, i.e., $\mathcal{B}_{k}=\mathcal{B} \forall k$, such that, each active user is 

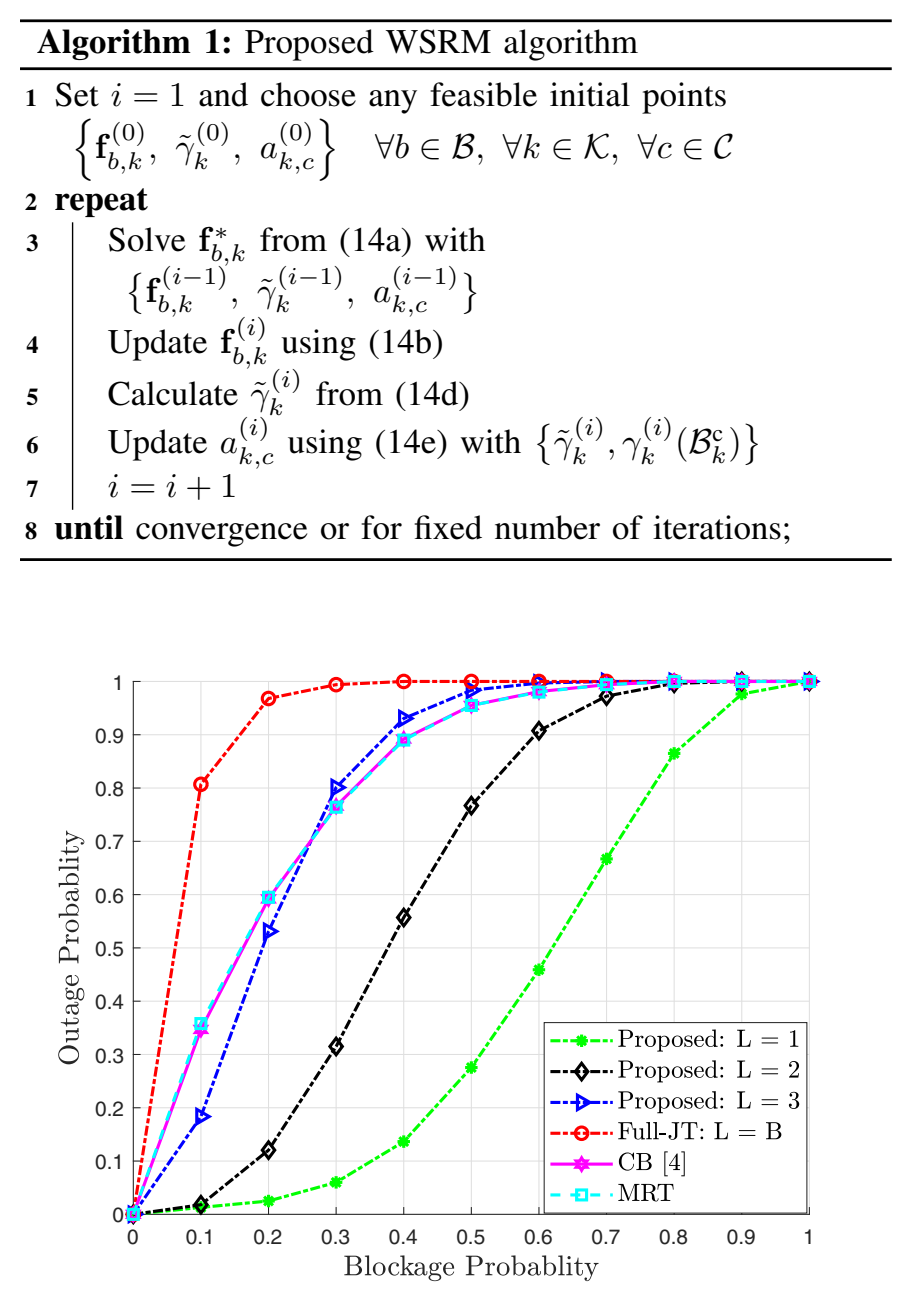

Fig. 2. Outage performance as function of increasing blockage probability.

coherently served by all BSs (excluding the potentially blocked links). For simplicity, all BSs are assumed with the same maximum transmit power, i.e., $P_{b}=P_{t} \forall b$, noise variance of $N_{o}=1$, carrier-frequency $f_{c}=28 \mathrm{GHz}$ and the cell-edge $\mathrm{SNR}=15 \mathrm{~dB}$. More precisely, SNR $=P_{t} / d_{e}^{-\varrho / 2} \forall b$, where $d_{e}^{-\varrho / 2}$ denotes the cell-edge distance and $\varrho=2$. The user priorities are set to be equal $\left(\delta_{k}=1 \forall k\right)$. All users are assumed to be randomly dropped within the coverage region, hence having different path gain and AoD with respect to each BS. If not mentioned otherwise, we use $\beta=0.005$ and $\psi=0.05$ for the subgradient and best response step sizes, respectively.

Outage event occurs if the assigned transmit rate $R_{k}$ exceeds the achievable link rate $C_{k}$, for any user $k=1, \ldots, K$, i.e., $P_{\text {out }} \triangleq \mathbf{P}\left\{R_{k}>C_{k}\right\} \forall k \in \mathcal{K}$. It can be concluded from Fig. 2 that the outage performance is greatly improved by decreasing the subset size $L$. Clearly, lower $L$ provides more stable and reliable communication. The beamformer design with $L=1$ can provide reliable connectivity even if all but one LoS links are blocked. For example, with link specific blockage probability 0.2 the outage probability is decreased from 0.9 to (close to) zero by changing $L$ from 4 to 1 . Thus, the specific allocation with $L=1$ can withstand blockage upto a single active link. Furthermore, it can be seen that the

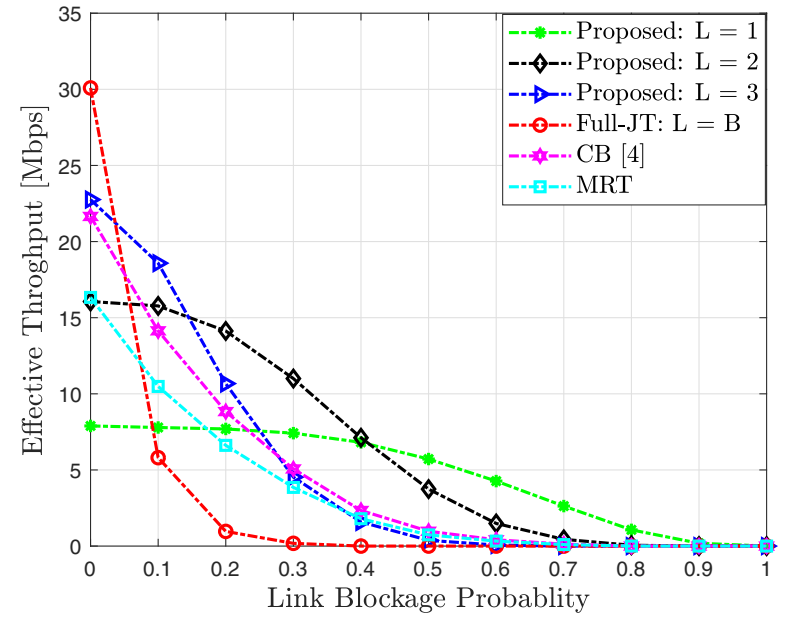

Fig. 3. Effective throughput with increasing blockage probability.

proposed method significantly outperforms the conventional JT $(L=B)$, Coordinated Beamforming (CB) and Maximum Ratio Transmission (MRT) based precoder design.

Fig. 3 illustrates the trade-off between achievable throughput and outage performance. Here, the effective throughput is defined as $T_{e} \triangleq\left(1-P_{\text {out }}\right) R$, where $R=\sum_{k \in \mathcal{K}} \log \left(1+\tilde{\gamma}_{k}\right)$, i.e., when each active user successfully receives transmit data. It can be observed that with the smaller subset size $L$, throughput is significantly reduced due to pessimistic estimate of the aggregated SINR. However, it remains stable at much higher link blockage probabilities. On the contrary, with the conventional JT $(L=B)$, the throughput quickly approaches zero due to high outage. Clearly, there is trade-off between achievable system throughput and outage performance. More specifically, for a given outage threshold, we can guarantee minimum achievable throughput and vice versa. Our proposed method provides more robust and resilient connectivity under uncertainties of mmWave channel, whereas, with the conventional JT, CB and MRT methods, sum-rate rapidly decreases towards zero if the blockage probability is slightly increased.

\section{CONCLUSION}

In this paper, we studied robust and reliable downlink transmission in mmWave wireless access via CoMP connectivity. We proposed a novel and computationally efficient iterative algorithm based on SCA framework and parallelization of the corresponding KKT solutions, while accounting for the uncertainties of mmWave channel in terms of link blockage. We devised a low complexity scheme, which is tractable for practical implementations, based on the best response and subgradient methods, wherein, each BS-specific variables are optimized in parallel. This provided significant reduction in computational complexity with respect to joint optimization over the BS specific precoders. Simulation results manifested the robustness of proposed beamformer design in presence of random link blockages. The outage performance and achievable sum-rate with the proposed method significantly outperform the baseline scenarios and results in a more stable connectivity for highly reliable communication. 


\section{APPENDIX A}

Considering the Lagrangian in (13), the KKT optimality conditions for the dual variables $a_{k, c}$ and $z_{b}$ are obtained by differentiating with respect to each associated primal optimization variables $\tilde{\gamma}_{k}$ and $\mathbf{f}_{b, k}$. After some algebraic manipulations, stationary conditions are given as

$$
\begin{aligned}
& \nabla_{\tilde{\gamma}_{k}}:-\frac{\delta_{k}}{1+\tilde{\gamma}_{k}}+\sum_{c \in \mathcal{C}} a_{k, c} \frac{N_{o}+\sum_{i \in \mathcal{K}}\left|\overline{\mathbf{h}}_{k}^{\mathrm{cH}} \overline{\mathbf{f}}_{i}^{(i)}\right|^{2}}{\left(1+\tilde{\gamma}_{k}^{(i)}\right)^{2}}=0 \\
& \nabla_{\mathbf{f}_{b, k}}: \mathbf{f}_{b, k}^{\mathrm{H}}\left(\mathbb{I} z_{b}+\sum_{c \in \mathcal{C}} \sum_{k^{\prime} \in \mathcal{K} \backslash k} a_{k^{\prime}, c} \mathbf{h}_{b, k^{\prime}}^{\mathrm{c}} \mathbf{h}_{b, k^{\prime}}^{\mathrm{cH}}\right)= \\
& \sum_{c \in \mathcal{C}} a_{k, c} \frac{\left(\overline{\mathbf{f}}_{k}^{(i) \mathrm{H}} \overline{\mathbf{h}}_{k}^{\mathrm{c}}\right)}{1+\tilde{\gamma}_{k}^{(i)}} \mathbf{h}_{b, k}^{\mathrm{cH}} \\
& \quad-\sum_{c \in \mathcal{C}} \sum_{k^{\prime} \in \mathcal{K} \backslash k} a_{k^{\prime}, c}\left(\sum_{b^{\prime} \in \mathcal{B} \backslash b} \mathbf{f}_{b^{\prime}, k}^{\mathrm{H}} \mathbf{h}_{b^{\prime}, k^{\prime}}^{\mathrm{c}} \mathbf{h}_{b, k^{\prime}}^{\mathrm{cH}}\right) .
\end{aligned}
$$

In addition to (15) and the feasibility constraints, the KKT conditions also include the complementary slackness conditions as

$$
\begin{gathered}
a_{k, c}\left\{I_{k}\left(\mathcal{B}_{k}^{\mathrm{c}}\right)-\mathcal{F}_{k}^{(i)}\left(c, \overline{\mathbf{f}}_{k}, \tilde{\gamma}_{k} ; \overline{\mathbf{f}}_{k}^{(i)}, \tilde{\gamma}_{k}^{(i)}\right)\right\}=0 \quad \forall(k, c), \\
z_{b}\left\{\sum_{k \in \mathcal{K}}\left\|\mathbf{f}_{b, k}\right\|^{2}-P_{b}\right\}=0 \quad \forall b .
\end{gathered}
$$

Lets assume the user-specific priority weights $\delta_{k}>0 \forall k$ and we know $\tilde{\gamma}_{k} \geq 0 \forall k$. Then, from (15a), we can observe that

$$
\frac{\delta_{k}}{1+\tilde{\gamma}_{k}}=\sum_{c \in \mathcal{C}} a_{k, c} \frac{N_{o}+\sum_{i \in \mathcal{K}}\left|\overline{\mathbf{h}}_{k}^{\mathrm{cH}} \overline{\mathbf{f}}_{i}^{(i)}\right|^{2}}{\left(1+\tilde{\gamma}_{k}^{(i)}\right)^{2}}>0 \quad \forall k .
$$

In other words, at least one of dual variables $a_{k, c} \forall c$ for each user $k$ is strictly positive and RHS of (17) is zero if and only if $\delta_{k}=0$. For simplicity, (17) can be rewritten as

$$
\tilde{\gamma}_{k}=\delta_{k}\left\{\sum_{c \in \mathcal{C}} a_{k, c} \frac{N_{o}+\sum_{i \in \mathcal{K}}\left|\overline{\mathbf{h}}_{k}^{\mathrm{cH}} \overline{\mathbf{f}}_{i}^{(i)}\right|^{2}}{\left(1+\tilde{\gamma}_{k}^{(i)}\right)^{2}}\right\}^{-1}-1 \forall k .
$$

The dual variables $a_{k, c} \forall c$ are highly coupled and interdependent due to common SINR constraint, as also seen from (15a) and (16a). Therefore, we cannot calculate the exact values of these variables in a closed-form expression. However, all the coupled non-negative Lagrangian multiplier $a_{k, c} \forall(k, c)$ can be iteratively solved using the subgradient method such as based on constrained ellipsoid method. For iteration $i$, the update for the dual variable $a_{k, c}$ with a small positive step size $\beta$ can be formulated as

$$
a_{k, c}^{(i)}=\left(a_{k, c}^{(i-1)}+\beta\left[\tilde{\gamma}_{k}^{(i)}-\gamma_{k}^{(i)}\left(\mathcal{B}_{k}^{\mathrm{c}}\right)\right]\right)^{+} \forall(k, c) .
$$

The dual variables $\mathbf{a}^{(0)}=\left[a_{1,1}^{(0)}, a_{2,1}^{(0)}, \ldots, a_{K, C}^{(0)}\right]^{\mathrm{T}}$ are initialized with positive small values. Finally, from $(15 b)$, we obtain the transmit precoder as in (14a). The dual variables $z_{b} \forall b$ are chosen to satisfy the total power constraints (16b), using the bisection search method. It should be noted, in the multi-cell scenario, the total sum-power constraint may not necessary hold with the equality. Specifically, for each BS $b$ if $\sum_{k \in \mathcal{K}}\left\|\mathbf{f}_{b, k}^{*}\right\|^{2}<P_{b}$ then $z_{b}=0$ i.e., non-negative dual variable $z_{b}$ is set zero in order to satisfy the corresponding complementary slackness condition [21]. Otherwise, there exist $z_{b}>0$ such that $\sum_{k \in \mathcal{K}}\left\|\mathbf{f}_{b, k}^{*}\right\|^{2}=P_{b}$, this value is unique and provide the optimal solution to the optimization problem [21], which can be easily found by using the bisection search with respect to the total sum power constraint.

\section{REFERENCES}

[1] T. S. Rappaport et al., "Millimeter wave mobile communications for 5G cellular: It will work!" IEEE Access, vol. 1, pp. 335-349, 2013.

[2] J. G. Andrews et al., "What will 5G be?" IEEE J. Sel. Areas Commun., vol. 32, no. 6, pp. 1065-1082, Jun. 2014.

[3] G. R. MacCartney et al., "Rapid fading due to human blockage in pedestrian crowds at 5G millimeter-wave frequencies," in Proc. IEEE Global Commun. Conf., Dec. 2017, pp. 1-7.

[4] A. Tölli, H. Pennanen, and P. Komulainen, "On the value of coherent and coordinated multi-cell transmission," in Proc. IEEE Int. Conf. Commun. Workshop, Jun. 2009, pp. 1-5.

[5] R. Irmer et al., "Coordinated multipoint: Concepts, performance, and field trial results," IEEE Commun. Mag., vol. 49, no. 2, pp. 102-111, Feb. 2011

[6] G. R. MacCartney and T. S. Rappaport, "Millimeter-wave base station diversity for 5G coordinated multipoint (CoMP) applications," IEEE Trans. Wireless Commun., vol. 18, no. 7, pp. 3395-3410, Jul. 2019.

[7] G. R. MacCartney, T. S. Rappaport, and A. Ghosh, "Base station diversity propagation measurements at $73 \mathrm{GHz}$ millimeter-wave for 5G coordinated multipoint (CoMP) analysis," in Proc. IEEE Global Commun. Conf. Workshops, Dec. 2017, pp. 1-7.

[8] D. Maamari, N. Devroye, and D. Tuninetti, "Coverage in mmWave cellular networks with base station co-operation," IEEE Trans. Wireless Commun., vol. 15, no. 4, pp. 2981-2994, Apr. 2016.

[9] 3GPP TSG RAN Meeting \#82, TS 37.340, "NR; Multi-connectivity; Overall description (Release 15),” Rel. 15, Jun 2018.

[10] J. Kaleva, A. Tölli, and M. Juntti, "Decentralized sum rate maximization with QoS constraints for interfering broadcast channel via successive convex approximation," IEEE Trans. Signal Process., vol. 64, no. 11, pp. 2788-2802, Jun. 2016.

[11] S. Boyd et al., "Subgradient methods," lecture notes of EE392o, Stanford University, 2003.

[12] J. Kaleva et al., "Decentralized joint precoding with pilot-aided beamformer estimation," IEEE Trans. Signal Process., vol. 66, no. 9, pp. 2330-2341, May 2018.

[13] A. Checko et al., "Cloud RAN for mobile networks-A technology overview," IEEE Communications Surveys Tutorials, vol. 17, no. 1, pp. 405-426, Firstquarter 2015.

[14] M. D. Renzo, "Stochastic geometry modeling and analysis of multitier millimeter wave cellular networks," IEEE Trans. Wireless Commun., vol. 14, no. 9, pp. 5038-5057, Sep. 2015.

[15] D. Kumar et al., "Reliable positioning and mmWave communication via multi-point connectivity," Sensors, vol. 18, no. 11, p. 4001, 2018

[16] G. Venkatraman et al., "Traffic aware resource allocation schemes for multi-cell MIMO-OFDM systems," IEEE Trans. Signal Process., vol. 64, no. 11, pp. 2730-2745, Jun. 2016.

[17] D. Kumar, J. Kaleva, and A. Tölli, "Multi-point connectivity for reliable mmWave," in Proc. European Sign. Process. Conf., Sep. 2019.

[18] J. Kaleva, R. Berry, M. Honig, A. Tölli, and M. Juntti, "Decentralized sum MSE minimization for coordinated multi-point transmission," in Proc. IEEE Int. Conf. Acoust., Speech, Signal Processing, May 2014, pp. 469-473.

[19] M. Grant and S. Boyd, "CVX: Matlab software for disciplined convex programming, version 2.1," http://cvxr.com/cvx, Mar. 2014.

[20] M. S. Lobo, L. Vandenberghe, S. Boyd, and H. Lebret, "Applications of second-order cone programming," Linear Algebra and Applications, vol. 284, pp. 193-228, Nov. 1998.

[21] S. Boyd and L. Vandenberghe, Convex optimization. Cambridge university press, 2004. 